\title{
Case Report: Motivational Interviewing to Reinforce Coping and Improve Quality of Life in Patients with Spinal Cord Injury
}

\author{
Maryam Buhari $^{1}$ (D), Auwal Abdullahi ${ }^{2^{*}}$ (D) \\ 1. Department of Physiotherapy, Sir Muhammad Sunusi Specialists Hospital, Kano, Nigeria. \\ 2. Department of Physiotherapy, Faculty of Allied Health Sciences, Bayero University, Kano, Nigeria.
}

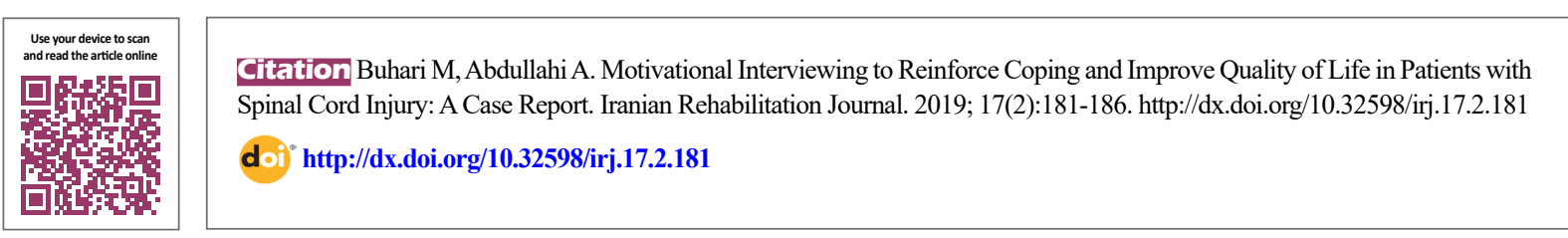

\section{(i) (8)}

Article info:

Received: 28 Aug 2018

Accepted: 10 Jan 2019

Available Online: 01 June 2019

\section{Keywords:}

Spinal Cord Injury, Coping strategies, Motivational interviewing, Quality of Life

\section{ABSTRACT}

Objectives: Spinal Cord Injury (SCI) can adversely affect ones' life and coping with it is very important. The present study reported the effects of reinforcing coping strategies using Motivational Interviewing (MI)

Methods: This was a case report of 5 males with SCI, with the Mean \pm SD age and time passed since the SCI of $34 \pm 15.9$ years and $20.6 \pm 16.6$, respectively. The study participants were examined as part of routine clinical services at the Physiotherapy Department of Aminu Kano Teaching Hospital. Fifteen minutes of MI was individually provided to the study participants two times a week and for three weeks. Data were collected using the American Spinal Cord Injury Association (ASCIA) impairment scale, COPE inventory, and Spinal Cord InjuryQuality of Life (SCI-QoL) questionnaire. The obtained data were analyzed using descriptive statistics.

Results: The obtained results revealed that all participants gained scores ranging from 3-4 on the satisfaction domain of quality of life at the baseline, which increased to 5 at the postintervention phase. For the importance domain of quality of life, all patients had a maximum score of 5 at the baseline, which remained unchanged at post-intervention.

Discussion: People living with SCI can benefit from MI in terms of improved coping strategies. However, these findings are limited due to the nature of the study design and the sample size. Therefore, more rigorous study designs are required to further investigate the findings of this study.

\section{* Corresponding Author:}




\section{Highlights}

- Patients with spinal cord injuries may suffer from depression and anxiety.

- Motivational interviewing can help people living with disability secondary to spinal cord injury to cope better with their condition and experience a better quality of life.

\section{Plain Language Summary}

Spinal Cord Injury (SCI) can result in a disability that shatters the dreams of the affected patient. SCI can affect movements of body parts such as the hands and the legs. As a result, the affected patients cannot effectively carry out their daily activities such as eating and walking. So the patients become partially or completely dependent on others for everyday activities. Such disabilities and dependence can lead to depression and possibly anxiety. However, some strategies can be used to improve coping abilities and quality of life in these patients. In this study, one strategy known as motivational interviewing (a discussion with the patients to help them overcome their problems and find solutions) is used for these patients. At the end of the study, the participants showed improvement in their coping ability and quality of life. These findings suggest that motivational interviewing can be used to help patients with SCI cope with their conditions and experience a better quality of life.

\section{Introduction}

pinal Cord Injury (SCI) often occurs unexpectedly. It may be caused as a result of falls, road traffic accidents, assaults, or pathological processes like the tuberculosis of the spine $[1,2]$. However, the major cause of SCI is road traffic accident around the world; in some quarters, it accounts for about $31 \%$ of all SCI cases [2]. Irrespective of the cause of SCI, it is usually accompanied by many complications, such as the loss of sensorimotor functions and the loss of urethral and anal control that could negatively affect patient's bio-psycho-psychological functions $[1,3]$.

The main physical functions affected following SCI are the basic and instrumental activities of daily living such as bathing and totaling, shopping , and managing finances. Thus, the affected people often have to depend on either formal or informal caregivers for daily activities. Depending on others for carrying out daily activities such as bathing, feeding and totaling may reduce self-esteem, and cause depression $[4,5]$.

They may also face problems with participation in previous leisure activities that used to bring them happiness and improved their Quality of Life (QoL). Consequently, people living with SCI may find it difficult to cope with their condition; therefore, they may need to develop some coping strategies. Coping strategy is a process of making behavioral and cognitive efforts to adapt to a stressful situation. The most common coping strategies are the acceptance of the situation, information seeking, positive reforming, religious beliefs, social support seeking, and self-trust $[6,7]$.
Following SCI, coping largely influences ones' living, the QoL, psychological and emotional stability, and adjustment [6]. Therefore, helping patients use positive coping strategies or reinforcing their positive coping strategies may reduce the use of negative coping strategies like substance use; and improve the QoL of people living with SCI. The present study aimed to use Motivational Interviewing (MI) to reinforce coping strategies and improve the QoL in people living with SCI. MI is a talk therapy aimed at improving patients' locus of control to positively cope with their conditions [8-10]. MI has previously been used to improve physical activity in people living with SCI [11]; and improve empathy and decrease burnout in professionals involved in the rehabilitation of this population [12].

\section{Methods}

The current case report study reported the effect of MI on reinforcing coping strategies and improving the QoL in people with SCI. The study participants included 5 males with the Mean \pm SD age and time passed since the SCI of $34 \pm 15.9$ years and $20.6 \pm 16.6$, respectively. The study participants were examined as part of routine clinical services rendered to SCI patients attending the Physiotherapy department at Aminu Kano Teaching Hospital.

The consents of the study participants were obtained for the publication of the progress of their conditions. This was achieved after explaining them about the benefits of doing so, in details. None of the study participants reported any previous history of other psychological interventions. 
Table 1. Characteristics of study participants

\begin{tabular}{|c|c|c|c|c|c|c|c|c|}
\hline Participant & Cause & Age (Years) & Sex & $\begin{array}{l}\text { Duration of } \\
\text { SCI (Months) }\end{array}$ & $\begin{array}{c}\text { Marital } \\
\text { Status }\end{array}$ & $\begin{array}{c}\text { Occupation } \\
\text { (Pre-SCl) }\end{array}$ & $\begin{array}{l}\text { Occupation } \\
\text { (Post-SCI) }\end{array}$ & $\begin{array}{c}\text { ASIA } \\
\text { Classification }\end{array}$ \\
\hline 1 & Gunshot & 47 & $M$ & 48 & Married & Retired & Unemployed & B \\
\hline 2 & RTA & 40 & $M$ & 24 & Married & Trading & Unemployed & A \\
\hline 3 & Assault & 16 & $M$ & 12 & Single & Student & Dropout & C \\
\hline 4 & RTA & 18 & $M$ & 7 & Single & Trading & Unemployed & A \\
\hline 5 & RTA & 49 & $M$ & 12 & Married & Driver & Unemployed & A \\
\hline
\end{tabular}

RTA: Road Traffic Accident

Iranian Rehabilitation Journal

The demographic characteristics of the study participants are described in Table 1.

Fifteen minutes of one-on-one MI were conducted with the samples in the presence of their caregivers, two times a week and for three weeks. The MI sessions involved discussion and providing information on coping strategies such as positive reforming, acceptance, social support seeking, religious beliefs, and problem-solving skills. Discussion on positive reforming was focused on helping patients see their condition as not preventing them from opportunities in life. They were assured that can achieve their goals in life, like any other person.

The discussions on acceptance was concentrated on making the participants understand the reality of their condition to become more aware of it and accept it. The discussion on social support seeking was focused on how the study participants could engage in social activities with their family and friends. The discussion on religious beliefs was focused on how the participants could use faith to accept and positively reform their condition. The discussion on problem-solving was consecrated on the analysis and evaluation of the study participants' problems, creating a solution and monitoring the effects of that solution.

The investigation tools used in the study included the American Spinal Cord Injury Association (ASCIA) impairment scale, COPE inventory, and Spinal Cord Injury-Quality of Life (SCI-QoL) questionnaire. The ASIA scale assesses the completeness of SCI by considering the key sensorimotor functions; it also investigates sparing in the lowest sacral segment, S4-S5 [13-15]. Pinprick and light touch were applied to test the sensory functions on different sensory areas, and dermatomes C2 to S5. These tests were scored on a 4-point scale, including 0-3 and not tested (in case of inability to test an area or dermatome). The motor function was examined by testing 10 different key muscles of the upper and lower limbs; it was scored on a 6-point scale, including $0-5$, not tested, and NT (in case of inability to test an area or dermatome).

ASIA Impairment Scale (AIS) classifies SCI into 5 classes, including A-E $[13,15]$. Class A comprises complete injury and indicates the complete absence of sensorimotor function in the sacral segments, S4-S5. Class B consists of incomplete sensory injury and reflects the presence of sensory function, but the complete absence of motor function below Neurological Level of Injury (NLI) and includes the sacral segments of S4-S5; moreover, no motor function is preserved more than three levels below the motor level on either side of the body.

Class $\mathrm{C}$ comprises incomplete motor injury and indicates the presence of motor function below the NLI of A, and more than half of key muscle functions below the single NLI have a muscle grade of $<3$ (grades $0-2$ ). Class D consists of incomplete, motor injury and demonstrates the presence of motor function below the NLI, and at least half (half or more) of key muscle functions below the NLI have a muscle grade of $>3$. Class E suggests normal sensorimotor function. The scale has been reported to be valid and reliable [14]

The COPE inventory is a valid and reliable tool that assesses individuals' coping strategies with stress [16-18]. This 63-item tool consists of 15 subclasses. The respondents are requested to rate themselves on a 4-point scale, as follows: 1. 'I usually don't do this at all'; 2 . 'I usually do this a little bit'; 3. 'I usually do this a medium amount'; and 4. 'I usually do this a lot' [16]. The SCI-QOL questionnaire has two subclasses, each including 37 items [19]. The first subclass asks a question, 'how satisfied are you with your life'; whereas the second subclass asks a question, 'how important to you is the area of your life?'. SCI-QOL is an appropriate tool for measuring the QoL in individuals with SCI [1].

In this study, the ASIA scale was used to classify the injury level of the study participants at the baseline; whereas the COPE inventory and the SCI-QOL were used to assess 
the study participants' coping abilities and QoL, respectively at the baseline and 3 weeks post-intervention. All of the obtained data were analyzed using descriptive statistics.

\section{Results}

The obtained results revealed that all participants achieved scores ranging from 3-4 on the satisfaction domain of the QoL at the baseline, which increased to 5 at the post-intervention phase. This indicates that the patients had an acceptance level of QoL on this domain at the baseline; however, it improved following the intervention to the extent that all the patients acquired a maximum score of 5 . However, for the importance domain of QoL, all patients gained a maximum score of 5 at the baseline which remained unchanged at the post-intervention phase. This suggests MI can help patients maintain emotional adjustment and the internal locus of control. Details of the obtained results are described in Table 2.

The COPE inventory data indicated improvements from the baseline to 3 weeks post-intervention; maintained or unchanged coping ability in some scales. These results suggest that to some extent, MI can help reinforce coping strategies in people living with SCI. Details of the obtained results are described in Table 3.

Table 2. Quality of life scores of the study participants pre and post intervention

\begin{tabular}{ccccc}
\hline Participants & HSQoL Pre & HSQoL Post & HSQoL Pre & HSQoL Post \\
\hline 1 & 4 & 5 & 5 & 5 \\
2 & 4 & 5 & 5 & 5 \\
3 & 4 & 5 & 5 & 5 \\
4 & 3 & 4 & 5 & 5 \\
\hline
\end{tabular}

HSQoL: How Satisfied domain of Quality of Life; HIQoL: How Important domain of Quality of Life

Table 3. Coping inventory pre and post motivational interviewing

\begin{tabular}{cccccccccccccccc}
\hline PT & PR1 & PR2 & MD1 & MD2 & FV1 & FV2 & ISS1 & ISS2 & AC1 & AC2 & DE1 & DE2 & RC1 & RC2 \\
\hline 1 & 2 & 4 & 2 & 3 & 3 & 2 & 2 & 4 & 4 & 4 & 1 & 2 & 4 & 4 \\
2 & 3 & 4 & 2 & 3 & 2 & 3 & 3 & 4 & 2 & 4 & 2 & 2 & 4 & 4 \\
\hline 3 & 2 & 4 & 1 & 4 & 3 & 2 & 2 & 4 & 4 & 4 & 2 & 3 & 4 & 4 \\
\hline 4 & 3 & 4 & 3 & 3 & 2 & 2 & 3 & 4 & 3 & 4 & 3 & 2 & 4 & 4 \\
\hline 5 & 2 & 4 & 3 & 2 & 3 & 2 & 4 & 3 & 4 & 4 & 3 & 3 & 4 & 4 \\
\hline HU1 & HU2 & BD1 & BD2 & RS1 & RS2 & ESS1 & ESS2 & SU1 & SU2 & AC1 & AC2 & SC1 & SC2 & PL1 & PL2 \\
\hline 1 & 3 & 2 & 2 & 3 & 3 & 3 & 4 & 1 & 1 & 2 & 4 & 4 & 1 & 3 & 4 \\
2 & 3 & 2 & 2 & 2 & 2 & 4 & 3 & 1 & 1 & 3 & 4 & 3 & 2 & 4 & 3 \\
\hline 1 & 3 & 2 & 2 & 2 & 3 & 2 & 4 & 1 & 1 & 2 & 3 & 3 & 2 & 3 & 3 \\
\hline 4 & 4 & 3 & 2 & 2 & 2 & 3 & 4 & 1 & 1 & 3 & 4 & 4 & 3 & 4 & 3 \\
\hline 2 & 3 & 3 & 2 & 3 & 2 & 3 & 4 & 1 & 1 & 2 & 4 & 4 & 2 & 3 & 4 \\
\hline
\end{tabular}

Iranian Rehabilitation Journal

Likert scale (1-4) Pt: Participant; PR: Positive Reframing/Reinforcement; MD: Mental Disengagement; FV: Focus on Venting of emotions; ISS-use of instrumental social support; AC: Active Coping; DE: Denial; RC: Religious Coping; HU: Humor; BD: Behavioural Disengagement; RS: Restraints; ESS: Emotional Social Support; SU: Substance Use; AC: Acceptance; SC: Suppression of Competing activities; PL: Planning 


\section{Discussion}

The present study aimed to report the effect of MI on reinforcing coping strategies and improving the $\mathrm{QoL}$ in patients with SCI. The obtained results suggested that MI improves or maintains coping abilities and the QoL in patients with SCI. Similarly, the use of positive coping strategies has been reported to have a positive influence on SCI patients [20,21]. Additionally, the positive influences may be correlated with each other. For instance, an appropriate coping behavior is well correlated with the QoL [22].

Individuals respond to stressful events in various ways and use different coping strategies. Moreover, coping has a positive relationship with the QoL; thus, the use of rehabilitation techniques that will help reinforce these strategies is important. One of these potential techniques is MI. MI has been used in SCI and other conditions like stroke to improve confidence and resolve patients' problems. It has been also applied to maximize achieving rehabilitation goals or independence in daily living activities $[9,10]$.

The technique can help improve compliance with exercise behaviors [23]. Improved exercise behavior is associated with bio-psychological benefits in healthy people and patients. Thus, MI targets behavior changes and self-motivational statements to enhance the process of positive change by the clients or patients [24,25]. The process of eliciting such positive change usually comprises 5 ways [26]. These ways include an expression of empathy through reflective listening, developing discrepancy between clients' goals or values and their current behavior, avoiding argument and direct confrontation, adjusting to client resistance rather than directly opposing it, and supporting self-efficacy and optimism.

The present study applied these ways in MI sessions to help the participants regain their internal locus of control; thus, they would better cope with their present conditions and be responsible for the quality of their lives. However, one of the limitations of this study was the lack of controls. Secondly, the sample size was very small and inadequate to be able to generalize the findings to all SCI patients.

\section{Conclusion}

MI seems to empower patients with SCI to regain their internal locus of control for better coping with their conditions and have improved QoL. However, these effects need to be further investigated using more rigorous research designs and a large sample size.

\section{Ethical Considerations}

\section{Compliance with ethical guidelines}

The study complied with the ethical guidelines of the hospital where the data was collected in terms of maintaining participants' privacy, seeking their consent and legal framework that enabled (MB) collected the data.

\section{Funding}

This research did not receive any specific grant from funding agencies in the public, commercial, or not-for-profit sectors.

\section{Authors' contributions}

All authors contributed in designing, running, and writing all parts of the research.

\section{Conflict of interest}

The authors declared no conflict of interest.

\section{Acknowledgments}

We would like to thank the staff of Physiotherapy Department at Aminu Kano Teaching Hospital for their help and assistance during the study.

\section{References}

[1] Tulsky DS, Kisala PA, Victorson D, Tate DG, Heinemann AW, Charlifue S, et al. Overview of Spinal Cord Injury, Quality of Life (SCI-QoL) measurement system. Journal of Spinal Cord. 2015; 38(3):257-69. [DOI:10.1179/2045772315Y.0000000023] [PMID] [PMCID]

[2] Chen Y, Tang Y, Vogel LC, DeVivo MJ. Causes of spinal cord injury. Topics in Spinal Cord Injury Rehabilitation. 2013; 19(1):1-8 [DOI:10.1310/sci1901-1] [PMID] [PMCID]

[3] Hou S, Rabchevsky AG. Autonomic consequences of spinal cord injury. Comprehensive Physiology. 2014; 4(4):1419-53. [DOI:10.1002/cphy.c130045]

[4] Kennedy P, Rogers BA. Anxiety and depression after spinal cord injury: A longitudinal analysis. Archives of Physical Medicine and Rehabilitation. 2000; 81(7):932-7. [DOI:10.1053/ apmr.2000.5580] [PMID]

[5] Van Leeuwen CM, Kraaijeveld S, Lindeman E, Post MW. Associations between psychological factors and quality of life ratings in persons with spinal cord injury: A systematic review. Spinal Cord. 2012; 50(3):174-84. [DOI:10.1038/sc.2011.120] [PMID] 
[6] Babamohamadi H, Negarandeh R, Dehghan-Nayeri N. Barriers to and facilitators of coping with spinal cord injury for Iranian patients: A qualitative study. Nursing \& Health Sciences. 2011; 13(2):207-15. [DOI:10.1111/j.1442-2018.2011.00602.x] [PMID]

[7] Bako Kwah S, Abdullahi A. Coping strategies in people with spinal cord injury: A qualitative interviewing. Iranian Rehabilitation Journal. 2018; 16(2):195-202. [DOI:10.32598/irj.16.2.195]

[8] Miller W, Rollnick S. Motivational interviewing. New York: Guilford Press; 2012

[9] Watkins CL, Auton MF, Deans CF, Dickinson HA, Jack CI Lightbody CE, et al. Motivational interviewing early after acute stroke: A randomized, controlled trial. Stroke. 2007; 8(3):1004-9. [DOI:10.1161/01.STR.0000258114.28006.d7] [PMID]

[10] Watkins CL, Wathan JV, Leathley MJ, Auton MF, Deans CF, Dickinson HA, et al. The 12-month effects of early motivational interviewing after acute stroke: A randomized controlled trial. Stroke. 2011; 42(7):1956-61. [DOI:10.1161/STROKEAHA.110.602227] [PMID]

[11] Nooijen CF, Stam HJ, Bergen MP, Bongers-Janssen HM, Valent L, van Langeveld S, et al. Act-active research group. A behavioural intervention increases physical activity in people with subacute spinal cord injury: A randomised trial. Journal of Physiotherapy. 2016; 62(1):35-41. [DOI:10.1016/j.jphys.2015.11.003] [PMID]

[12] Lusilla-Palacios P, Castellano-Tejedor C. Training a spinal cord injury rehabilitation team in motivational interviewing. Rehabilitation Research and Practice. 2015; 2015(358151):1-7. [DOI:10.1155/2015/358151] [PMID] [PMCID]

[13] Hales M, Biros E, Reznik JE. Reliability and validity of the sensory component of the International Standards for Neurological Classification of Spinal Cord Injury (ISNCSCI): A systematic review. Topics in Spinal Cord Injury Rehabilitation. 2015; 21(3):2419. [DOI:10.1310/sci2103-241] [PMID] [PMCID]

[14] Furlan JC, Felilings MG, Tafor CH, Davis AM. Motor and sensory assessment of patients in clinical trials for pharmacological therapy in acute spinal cord injury; psychometric properties of ASIA standards. Journal of Neurotrauma. 2008; 25(11):1273-301. [DOI:10.1089/neu.2008.0617] [PMID]

[15] Kirshblum SC, Burns SP, Biering-Sorensen F, Donovan W, Graves DE, Jha A, et al. International standards for neurological classification of spinal cord injury (revised 2011). The Journal of Spinal Cord Medicine. 2011; 34(6):535-46. [DOI:10.1179/20457721 1X13207446293695] [PMID] [PMCID]

[16] Carver CS, Scheier MF, Weintraub JK. Assessing coping strategies: A theoretically based approach. Journal of Personality and Social Psychology. 1989; 56(2):267-83. [DOI:10.1037//00223514.56.2.267] [PMID]

[17] Donoghue KJ. Measuring coping: Evaluating the psychometric properties of the COPE [MA. Thesis]. Perth, Western Australia: Cowan University; 2004

[18] Litman JA. The COPE inventory: Dimensionality and relationships with approach-and avoidance-motives and positive and negative traits. Personality and Individual Differences. 2006; 41(2):273-84. [DOI:10.1016/j.paid.2005.11.032]

[19] Ferrans CE, Powers MJ. Quality of life index: Development and psychometric properties. Advances in Nursing Science. 1985; 8(1):15-24. [PMID]
[20] Kennedy P, Lude P, Elfström ML, Smithson E. Appraisals, coping and adjustment pre and post SCI rehabilitation: A 2-year follow-up study. Spinal Cord. 2012; 50(2):112-8. [DOI:10.1038/ sc.2011.127] [PMID]

[21] Dorsett P, Geraghty T, Sinnott A, Acland R. Hope, coping and psychosocial adjustment after spinal cord injury. Spinal Cord Series and Cases. 2017; 3:17046. [DOI:10.1038/scsandc.2017.46] [PMID] [PMCID]

[22] Elfstrom M, Rydén A, Kreuter M, Taft C, Sullivan M. Relations between coping strategies and health-related quality of life in patients with spinal cord lesion. Journal of Rehabilitation Medicine. 2005; 37(1):9-16. [DOI:10.1080/16501970410034414] [PMID]

[23] Rollnick S, Miller WR, Butler C. Motivational interviewing in health care: Helping patients change behavior. New York: Guilford Press; 2008.

[24] Davidson, R. Can psychology make sense of change? In: Edwards G, and Lader, M editors. Addiction: Processes of Change. Society for the Study of Addiction Monograph No. 3. New York: Oxford University Press; (1994).

[25] Miller WR, Rollnick S. Motivational interviewing: Preparing people to change addictive behavior. New York: Guilford Press; 1991.

[26] Center for Substance Abuse Treatment. Enhancing motivation for change in substance abuse treatment. In: Professional Care Providers, editor. TIP 35: Enhancing Motivation for Change in Substance Abuse Treatment. Rockville, Maryland: SAMHSA; 2013 